Supporting Information

\title{
The role of charge in lipid vesicle binding and vesicle surface saturation by Gaduscidin-1 and Gaduscidin-2
}

\author{
Gagandeep Sandhu†, Valerie Booth $¥ \dagger$, and Michael R. Morrow†* \\ $\dagger$ Department of Physics and Physical Oceanography and $\ddagger$ Department of Biochemistry, \\ Memorial University of Newfoundland, \\ St. John's, Newfoundland and Labrador, Canada, A1B 3X7
}

For cationic peptides binding to vesicles comprising a mixture of anionic and zwitterionic lipids, $\zeta$-potential is expected to increase linearly with total peptide concentration, $[\mathrm{P}]_{\mathrm{T}}$, at low peptide concentration $^{1}$ but reach a limiting value at high $[\mathrm{P}]_{\mathrm{T}}$ due to peptide saturation of the vesicle surface. This behavior is illustrated schematically in Figure S1 where $\zeta_{\max }$ is the limiting value of $\zeta$-potential at high peptide concentration for which the vesicle surfaces are peptide-saturated. $\zeta_{0}$ is the $\zeta$-potential of the lipid vesicles in the absence of peptide.

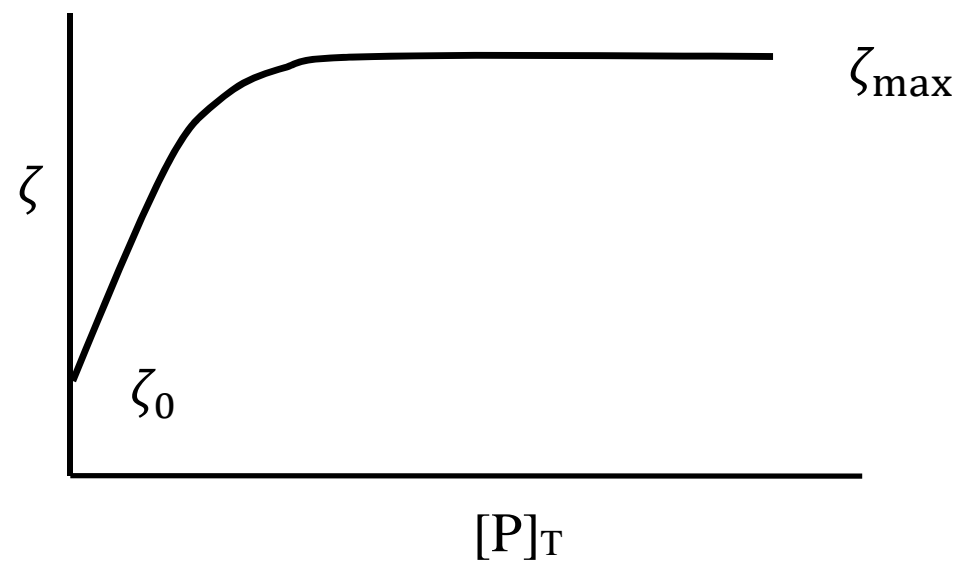

Figure S1. Schematic representation of the peptide concentration dependence of $\zeta$-potential for cationic peptides interacting with vesicles comprising anionic and zwitterionic lipids. $\zeta_{\max }$ is the limiting value of $\zeta$-potential at high peptide concentration for which the vesicle surfaces are peptidesaturated. $\zeta_{0}$ is the $\zeta$-potential of the lipid vesicles in the absence of peptide. 
In the discussion that follows, $V_{\mathrm{T}}$ denotes the total sample volume and $[\mathrm{P}]_{\mathrm{W}}$ is the concentration of peptide dissolved in water. It is also helpful to define $n_{\mathrm{T}}$ as the total number of peptide molecules in the sample, $n_{\mathrm{P}, \text { ad }}$ as the number of peptide molecules adsorbed to vesicle surfaces, and $n_{\mathrm{P}, \mathrm{W}}$ as the number of peptide molecules dissolved in the surrounding water. The total number of peptides can then be expressed as

$$
n_{\mathrm{P}, \mathrm{T}}=[\mathrm{P}]_{\mathrm{W}} V_{\mathrm{T}}+n_{\mathrm{P}, \mathrm{ad}}
$$

so that the total peptide concentration becomes

$$
[\mathrm{P}]_{\mathrm{T}}=[\mathrm{P}]_{\mathrm{W}}+\frac{n_{\mathrm{P}, \mathrm{ad}}}{V_{\mathrm{T}}} .
$$

The fraction of peptide molecules bound to vesicles is defined as

$$
X=\frac{n_{\mathrm{P}, \mathrm{ad}}}{n_{\mathrm{P}, \mathrm{T}}} .
$$

In the low peptide concentration regime, where $\zeta$ varies linearly with $[\mathrm{P}]_{\mathrm{T}}$, the fraction of peptide molecules bound to lipid vesicles is expected to be constant and will be denoted as $X_{0}$ here. This corresponds to $X_{L}$ in the notation of Freire et al. ${ }^{1}$ At high peptide concentration, for which the vesicle surfaces are saturated with bound peptide, $X$ is expected to decrease with increasing $[\mathrm{P}]_{\mathrm{T}}$.

It is expected that the maximum number of peptides that can be accommodated on vesicle surfaces will be proportional to the total number of lipid molecules, $n_{\mathrm{L}}$, in the sample. A proportionality factor $Q$ can be defined such that maximum number of peptides that can be accommodated on vesicles surfaces is then $Q n_{\mathrm{L}}$. If the vesicle surfaces are not saturated, then the number of additional peptides that can be accommodated is $Q n_{\mathrm{L}}-n_{\mathrm{P}, \mathrm{ad}}$. This number can also be thought of as an effective number of available (unoccupied) peptide binding sites on the vesicle surfaces. Since one bound peptide is expected to interact with multiple lipid molecules, $Q$ is expected to be less than unity and $Q^{-1}$ is, in effect, the number of lipids that interact with a given bound peptide.

If the total lipid concentration in a sample is denoted as $[\mathrm{L}]=n_{\mathrm{L}} / V_{\mathrm{T}}$, then the ratio between the total number of lipid molecules and the total number of bound peptide molecules can be written as

$$
\frac{n_{\mathrm{L}}}{n_{\mathrm{P}, \mathrm{ad}}}=\frac{[\mathrm{L}]}{X[\mathrm{P}]_{\mathrm{T}}} .
$$

In the limits of low and high peptide concentration, respectively, this ratio becomes 


$$
\lim _{[\mathrm{P}]_{\mathrm{T}} \rightarrow 0} \frac{n_{\mathrm{L}}}{n_{\mathrm{P}, \mathrm{ad}}}=\frac{[\mathrm{L}]}{X_{0}[\mathrm{P}]_{\mathrm{T}}}
$$

and

$$
\lim _{[\mathrm{P}]_{\mathrm{T}} \rightarrow \infty} \frac{n_{\mathrm{L}}}{n_{\mathrm{P}, \mathrm{ad}}}=Q^{-1}
$$

An equilibrium constant for peptide binding to vesicles can be defined by assuming that, at equilibrium, the rates of peptide adsorption and desorption are equal and then solving for the ratio of the corresponding rate constants. In analogy with the Langmuir model derivation of an adsorption isotherm ${ }^{2-4}$, we assume that the rate of peptide adsorption is proportional to the concentration of dissolved peptide, $[\mathrm{P}]_{\mathrm{W}}$, and the surface density of available peptide binding "sites" and that the rate of peptide desorption is proportional to the surface density of occupied peptide binding "sites". The rates of adsorption and desorption, respectively, can then be written as

$$
r_{\mathrm{ad}}=k_{\mathrm{ad}}[\mathrm{P}]_{\mathrm{W}} \frac{\left(Q n_{\mathrm{L}}-n_{\mathrm{P}, \mathrm{ad}}\right)}{A_{\mathrm{Ves}}}
$$

and

$$
r_{\mathrm{des}}=k_{\mathrm{des}} \frac{n_{\mathrm{P}, \mathrm{ad}}}{A_{\mathrm{Ves}}}
$$

where $k_{\text {ad }}$ and $k_{\text {des }}$ are rate constants for adsorption and desorption respectively and $A_{\text {Ves }}$ is the (unknown) total vesicle surface area.

At equilibrium, we expect $r_{\mathrm{ad}}=r_{\mathrm{des}}$ so that

$$
k_{\mathrm{ad}}[\mathrm{P}]_{\mathrm{W}}\left(Q n_{\mathrm{L}}-n_{\mathrm{P}, \mathrm{ad}}\right)=k_{\mathrm{des}} n_{\mathrm{P}, \mathrm{ad}}
$$

In further analogy with the Langmuir model for adsorption, an equilibrium constant for peptide adsorption can then be expressed as

$$
K_{\mathrm{eq}} \equiv \frac{k_{\mathrm{ad}}}{k_{\mathrm{des}}}=\frac{n_{\mathrm{P}, \mathrm{ad}}}{[\mathrm{P}]_{\mathrm{W}}\left(Q n_{\mathrm{L}}-n_{\mathrm{P}, \mathrm{ad}}\right)}
$$

which can be rearranged to give

$$
\frac{n_{\mathrm{P}, \mathrm{ad}}}{Q n_{\mathrm{L}}}=\frac{K_{\mathrm{eq}}[\mathrm{P}]_{\mathrm{W}}}{1+K_{\mathrm{eq}}[\mathrm{P}]_{\mathrm{W}}} .
$$


In Eq. S11, the left had side is, in effect, the fraction of available peptide binding sites that are occupied. It should be noted that the right hand side of Eq. S11 is expressed in terms of $[\mathrm{P}]_{\mathrm{W}}$ rather than the total peptide concentration.

In order to express $\zeta$-potential as a function of $[\mathrm{P}]_{\mathrm{T}}$, we follow Freire et al. ${ }^{1}$ and assume that each adsorbed peptide of charge $z_{\mathrm{P}}$ neutralizes a corresponding negative charge on a vesicle surface and that changes in $\zeta$-potential are proportional to changes in net charge on the vesicle surfaces. In particular, we assume that the change in $\zeta$-potential, $\Delta \zeta \equiv \zeta-\zeta_{0}$, is proportional to the number of adsorbed peptides so that

$$
\Delta \zeta \propto n_{\mathrm{P}, \mathrm{ad}}
$$

The maximum change in $\zeta$-potential, $\Delta \zeta_{\max } \equiv \zeta_{\max }-\zeta_{0}$, will then be proportional to the maximum number of peptides that can be accommodated on vesicle surfaces so that

$$
\Delta \zeta_{\max } \propto Q n_{\mathrm{L}}
$$

Accordingly,

$$
\frac{n_{\mathrm{P}, \mathrm{ad}}}{Q n_{\mathrm{L}}}=\frac{\Delta \zeta}{\Delta \zeta_{\max }}
$$

so that

$$
\frac{\Delta \zeta}{\Delta \zeta_{\max }}=\frac{K_{\mathrm{eq}}[\mathrm{P}]_{\mathrm{W}}}{1+K_{\mathrm{eq}}[\mathrm{P}]_{\mathrm{W}}}
$$

Using equations (S2), (S3), and (S4), it is possible to obtain two expressions for the dependence of $\zeta$-potential on peptide concentration. These are

$$
\zeta=\zeta_{0}+\Delta \zeta_{\max } \frac{K_{\mathrm{eq}}(1-X)[\mathrm{P}]_{\mathrm{T}}}{1+K_{\mathrm{eq}}(1-X)[\mathrm{P}]_{\mathrm{T}}}
$$

and

$$
\zeta=\zeta_{0}+\Delta \zeta_{\max } \frac{X[\mathrm{P}]_{\mathrm{T}}}{Q[\mathrm{~L}]}
$$

In Eqs. S16 and S17, the fraction of peptide bound to vesicle surfaces, $X$, is independent of peptide concentration for small $[\mathrm{P}]_{\mathrm{T}}$ but decreases with increasing peptide concentration for large $[\mathrm{P}]_{\mathrm{T}}$. For small peptide concentrations, $\zeta$ is expected to increase linearly with $[\mathrm{P}]_{\mathrm{T}}$ and Eqs. S16 and S17 become, respectively,

$$
\zeta=\zeta_{0}+\Delta \zeta_{\max } K_{\mathrm{eq}}\left(1-X_{0}\right)[\mathrm{P}]_{\mathrm{T}}
$$


and

$$
\zeta=\zeta_{0}+\Delta \zeta_{\max } \frac{X_{0}[\mathrm{P}]_{\mathrm{T}}}{Q[\mathrm{~L}]}
$$

The slope of $\zeta$ versus $[\mathrm{P}]_{\mathrm{T}}$ at small peptide concentration can thus be written in terms of $\Delta \zeta_{\max }$, $X_{0}$, and either $K_{\mathrm{eq}}$ or $Q$ so that

$$
\lim _{[\mathrm{P}]_{\mathrm{T}} \rightarrow 0} \frac{d \zeta}{d[\mathrm{P}]_{\mathrm{T}}}=\Delta \zeta_{\mathrm{max}} K_{\mathrm{eq}}\left(1-X_{0}\right)
$$

or

$$
\lim _{[\mathrm{P}]_{\mathrm{T}} \rightarrow 0} \frac{d \zeta}{d[\mathrm{P}]_{\mathrm{T}}}=\Delta \zeta_{\max } \frac{X_{0}}{Q[\mathrm{~L}]}
$$

An expression for $Q$ can be considering comparing the total net charge on the vesicles in the absence of peptide and when the vesicle surfaces are saturated with bound peptide. If the charge on a peptide is $Z_{\mathrm{P}}$ and the average lipid charge in the vesicles is $\overline{Z_{\mathrm{L}}}$ then the total net charge will be $n_{\mathrm{P}, \mathrm{ad}} z_{\mathrm{P}}+n_{\mathrm{L}} \overline{\mathrm{L}}$ for the general case and $n_{\mathrm{L}} \overline{Z_{\mathrm{L}}}$ in the absence of peptides. These cases correspond to $\zeta$ and $\zeta_{0}$ respectively so we expect

$$
\frac{\zeta}{\zeta_{0}}=\frac{n_{\mathrm{P}, \mathrm{ad}} z_{\mathrm{P}}+n_{\mathrm{L}} \overline{z_{\mathrm{L}}}}{n_{\mathrm{L}} \overline{\overline{\mathrm{L}}_{\mathrm{L}}}}
$$

At high peptide concentration, for which the vesicle surfaces are saturated by bound peptide, the total number of bound peptides is $Q n_{\mathrm{L}}$ and the $\zeta$-potential is $\zeta_{\max }$ so

$$
\frac{\zeta_{\max }}{\zeta_{0}}=\frac{Q z_{\mathrm{P}}+\overline{z_{\mathrm{L}}}}{\overline{z_{\mathrm{L}}}}
$$

which can be rearranged to give

$$
Q=\frac{\overline{z_{\mathrm{L}}}}{Z_{\mathrm{P}}} \frac{\Delta \zeta_{\mathrm{max}}}{\zeta_{0}}
$$

Eq. S24 can thus be used to obtain $Q$ from $\Delta \zeta_{\max }$ and $\zeta_{0}$. Eqs. S21 and S22 can then be used to obtain $X_{0}$ and $K_{\text {eq }}$ from the peptide concentration dependence of $\zeta$ in the low $[\mathrm{P}]_{\mathrm{T}}$ regime where $\zeta$ increases linearly with $[\mathrm{P}]_{\mathrm{T}}$.

Peptide adsorption as inferred from the peptide concentration dependence of $\zeta$-poential measurements has previously been interpreted ${ }^{1}$ in terms of a partition constant, 


$$
K_{\mathrm{p}}=\frac{[\mathrm{P}]_{\mathrm{L}}}{[\mathrm{P}]_{\mathrm{W}}}
$$

where $[\mathrm{P}]_{\mathrm{L}}=n_{\mathrm{P}, \text { ad }} / \mathrm{V}_{\mathrm{L}}$ is the concentration of peptide present in the lipid environment and $\mathrm{V}_{\mathrm{L}}$ is the volume of the lipid bilayer environment. The partition constant defined in this way is the ratio of peptide concentration in the lipid environment to peptide concentration in the water environment. It is apparent that $K_{\mathrm{P}}$ is constant at low total peptide concentration but must decrease with increasing peptide concentration once the lipid environment is saturated with peptide. Isolating $n_{\mathrm{P}, \mathrm{ad}} /[\mathrm{P}]_{\mathrm{W}}$ from Equations $\mathrm{S} 21$ and $\mathrm{S} 25$, allows $K_{\mathrm{P}}$ to be related to $K_{\mathrm{eq}}$ through

$$
K_{\mathrm{p}}=\frac{1}{V_{\mathrm{L}}} \times \frac{K_{\mathrm{eq}} Q n_{\mathrm{L}}}{1+K_{\mathrm{eq}}[\mathrm{P}]_{\mathrm{W}}} .
$$

In the limit of low peptide concentration, the partition constant can be expressed as

$$
K_{\mathrm{p} 0}=\frac{K_{\mathrm{eq}} Q n_{\mathrm{L}}}{V_{\mathrm{L}}} .
$$

As noted above, the fraction of peptide molecules associated with the lipid vesicles is $X$ and the limit of this fraction at low total peptide concentration, $X_{0}$, is also used to characterize peptide adsorption. The value of $X_{0}$ can be obtained by analysing the slope of $\zeta$-potential versus peptide concentration in the limit of low peptide concentration ${ }^{1}$. Because $K_{\text {eq }}$ depends on the capacity of the surface to accommodate additional peptides as well as on the number of adsorbed peptides and the concentration of free peptide, the relationship between $X$ and $K_{\text {eq }}$ is not linear. To see how $X$ and $K_{\text {eq }}$ are related, the substitutions, $n_{\mathrm{P}, \mathrm{ad}}=X n_{\mathrm{P}, \mathrm{T}}$ and $[\mathrm{P}]_{\mathrm{W}}=\left((1-X) n_{\mathrm{P}, \mathrm{T}}\right) / V_{\mathrm{T}}$ can be used to express the equilibrium binding constant as

$$
K_{\mathrm{eq}}=\frac{X}{Q[\mathrm{~L}](1-X)\left(1-\frac{X n_{\mathrm{P}, \mathrm{T}}}{Q n_{\mathrm{L}}}\right)}
$$

where $[\mathrm{L}]=n_{\mathrm{L}} / V_{\mathrm{T}}$ is the total lipid concentration. Applying the low peptide concentration limit to the right-hand side of Equation S28 gives

$$
K_{\mathrm{eq}}=\frac{X_{0}}{Q[\mathrm{~L}]\left(1-X_{0}\right)} .
$$

It can be seen that there is a non-linear relation between $K_{\mathrm{eq}}$ and $X_{0}$ and that the constraint also depends on $Q$. 


\section{References}

1. Freire, J. M.; Domingues, M. M.; Matos, J.; Melo, M.N.; Veiga, A. S.; Santos, N. C.; Castanho, M. A. R. B. Using zeta-potential measurements to quantify peptide partition to lipid membranes. Eur. Biophys. J. 2011, 40, 481-487.

2. Langmuir, I. The adsorption of gases on plane surfaces of glass, mica, and platinum. J. Am. Chem. Soc. 1918, 40, $1361\{1403$.

3. Smejtek, P.; Wang, S. Domains and anomalous adsorption isotherms of diplamitoylphosphatidylcholine membranes and lipophilic ions: pentacholrophenolate, tetraphenylborate, and dipicrylamine. Biophys. J. 1991, 59, 1064\{1073.

4. Smejtek, P.; Blochel, A.; Wang, S. Hydrophobicity and sorption of chlorophenolates to lipid membranes. Chemosphere 1996, 33, $177\{201$. 